\title{
Trinta anos de reabilitação cognitiva com o apoio do computador: o que a neuropsicologia tem a dizer?
}

\author{
Gérson Silva Santos Neto ${ }^{1}$ \\ Mariângela Santos de Jesus ${ }^{2}$ \\ Silvana Batista Gaino ${ }^{2}$
}

\begin{abstract}
Resumo: O modelo neuropsicológico permite explicar as alterações comportamentais e cognitivas de cada paciente, constituindo assim um diagnóstico cientificamente fundamentado, o qual constitui uma contribuição valiosa para o diagnóstico neurológico. Além disso, permite estabelecer sobre bases científicas um programa de reabilitação apoiado na identificação do componente do sistema que apresenta o déficit e o estado dos demais componentes do sistema suscetíveis a participar do processo de reabilitação. O computador, desde o final do séc. XX, tornou-se o instrumento mais adequado para auxiliar qualquer processo de reabilitação cognitiva e é extremamente útil para uma intervenção neuropsicológica, pois é capaz de reunir todas as ferramentas necessárias para a apresentação de situações da vida diária e exercícios mais interativos. Neste artigo, foi realizada uma busca nos últimos trinta anos das publicações sobre reabilitação neuropsicológica computadorizada, apresentando a pouca evolução dessa área, mesmo com o avanço das novas tecnologias.
\end{abstract}

Palavras-chave: Interação humano-computador. Reabilitação computadorizada. Reabilitação neuropsicológica.

\begin{abstract}
The neuropsychological model allows to explain the behavioral and cognitive changes of each patient, thus constituting a diagnosis scientifically based. This diagnosis as well grounded is a valuable contribution to the neurologic diagnosis. Moreover, it allows to establish scientific bases on a rehabilitation program based on the identification of the component system that features the deficit and the state of other system components susceptible to participate in the rehabilitation process. The computer since the end of the century. XX was constituted as the most appropriate instrument to assist any process of cognitive rehabilitation and extremely useful for a neuropsychological intervention, it is able to gather all the necessary tools for presenting everyday situations and exercises more interactive. In this paper we performed a search in the last 30 years of the publications on computerized neuropsychological rehabilitation, presenting little progress in this area even with the advancement of new technologies.
\end{abstract}

Keywords: Human-computer interaction. Computadorized rehabilitation. Neuropsychology rehabilitation.

\section{Introdução}

Avanços tecnológicos vêm possibilitando um aumento da taxa de sobrevivência de pessoas vítimas de diferentes lesões neurológicas, bem como reduções significativas nos déficits cognitivos e/ou comportamentais. O processo conhecido por corrigir/minimizar os efeitos genéricos de déficits, de forma que sejam encontrados os

\footnotetext{
${ }^{1}$ Departamento de Neurociências e Ciências do Comportamento, USP-RP, Av. Bandeirantes, 3900 - Ribeirão Preto(SP) Brasil.

\{gerson.neto@msn.com\}

${ }^{2}$ Curso de Psicologia, UFRB, CCS - Avenida Carlos Amaral, 1015 - Santo Antônio de Jesus (BA) - Brasil.

\{mariangela.santos@msn.com, silgaino@hotmail.com\}
}

http://dx.doi.org/10.5335/rbca.2014.3259

Revista Brasileira de Computação Aplicada (ISSN 2176-6649), Passo Fundo, v. 6, n. 1, p. 60-70, abr. 201460 
meios adequados para alcançar metas funcionais específicas é chamado de reabilitação cognitiva. Essa disciplina, mesmo havendo relatos de reabilitação desde 400 a. c. [7], somente começou a ser investigada com mais atenção após a primeira guerra mundial [50]. Essas investigações permitiram concluir que a melhora atribuída à reabilitação pode ser separada do progresso no funcionamento que ocorre espontaneamente. No entanto, foi a partir da segunda revolução cognitiva na década de 1980 que a investigação de sequelas de lesões encefálicas tornou-se sistemática.

Paralelamente a esse aumento de trabalhos envolvendo a reabilitação cognitiva, as tecnologias da informática estavam sendo vistas como uma potente ferramenta para o auxílio no tratamento de saúde, aparecendo técnicas de diagnóstico por imagens muito mais acuradas e rápidas. Percebendo esse potencial, Bracy, em 1983, desenvolveu uma série de pesquisas apresentando as vantagens da utilização do computador para a reabilitação cognitiva, dando ênfase às ilimitadas possibilidades oferecidas pelo ambiente virtual. Desse modo, parecia claro que o futuro da reabilitação de funções cognitivas e comportamentais dependia da junção de conhecimentos relativos às injúrias e da adequação da tecnologia digital para esse fim. O foco deste trabalho situa-se em um estudo de revisão sistemática da literatura dos últimos trinta anos em que o uso do computador vem auxiliando o processo de reabilitação, tanto cognitiva, quanto neuropsicológica, sendo necessárias antes algumas considerações.

\subsection{Neuropsicologia clássica e a neuropsicologia cognitiva: qual a diferença?}

Fruto da inexistência de técnicas que permitissem observar o funcionamento cerebral dos seres vivos no século XIX e da inviabilidade do estudo mais direto das consequências de lesões cerebrais no mesmo período, salvo em circunstâncias excepcionais, é que se fundamentou a neuropsicologia. Nos seus primórdios, essa ciência estava preocupada em encontrar um método que auxiliasse na localização de lesões cerebrais a partir de análise das alterações comportamentais, cognitivas e emocionais. Eis o primeiro método neuropsicológico, o "método da correlação clínico-anatômica", no entanto, os primeiros neuropsicólogos não se contentaram em constatar e descrever as relações entre essas alterações cognitivo-comportamentais e as lesões das quais eram decorrentes. Por isso, tentaram explicar, muitas vezes com meios rudimentares, por que essas consequências eram presentes nesses casos [2].

Até a metade do séc. XX, a psicologia ainda não dispunha de um marco teórico para o estudo das alterações causadas por lesões cerebrais. Bruner foi o primeiro estudioso que para tentar sanar essa carência idealizou uma explicação por meio de modelos constituídos por "centros", cada centro seria responsável por um componente da função cognitiva afetada, e as vias nervosas que conectavam esses centros [1]. Esses modelos, considerados os precursores dos fluxogramas atuais, os quais utilizam o processamento de informação da neuropsicologia cognitiva, implicam que as funções cognitivas são dissociáveis e estão constituídas por componentes também dissociáveis.

A neuropsicologia cognitiva surgiu a partir da fusão da neuropsicologia com as ciências cognitivas, concebendo o cérebro como um sistema de processamento de informações e gerando modelos desse processamento para cada função cognitiva. Tais modelos permitem predizer determinadas alterações comportamentais em caso de lesão de algum dos seus componentes. Os processos de processamento de informação oferecem ao neuropsicólogo a possibilidade de formular hipóteses que guiem as suas avaliações e expliquem (e não apenas descrevam) os resultados.

A área de neuropsicologia cresceu exponencialmente nas últimas duas décadas, juntamente com outras áreas de pesquisa ligadas à neurociência [3]. A ênfase da neuropsicologia humana engloba os indivíduos com as mais diferentes disfunções cerebrais, desordens psiquiátricas e desordens afetivas, que podem ser resultado de uma ampla gama de circunstâncias: acidentes de parto, acidentes vasculares, quedas, acidentes esportivos, esquizofrenia, ou ainda desordens neurológicas causadas por paralisia cerebral, mal de Alzheimer, mal de Parkinson, esclerose múltipla, síndromes genéticas e tantas outras.

Nos mais jovens, as deficiências são associadas a vários problemas de desenvolvimento, falta de atenção causada por hiperatividade (Attention-deficit hyperactive disorder - ADHD) ou problemas de aprendizagem. Em um sentido amplo, a neuropsicologia é a ciência aplicada que verifica como as atividades específicas do cérebro são expressas em comportamentos observáveis [4]. Assim, apesar da importância da localização anatômica das lesões (neuropsicologia clássica), a visão holística da neuropsicologia propõe que o processamento mental ocorre em paralelo com subsistemas ou módulos que não são necessariamente relacionados a áreas anatômicas. A 
interrupção da coordenação desses módulos pode resultar no surgimento de um baixo nível de atividade de percepção ou ação. Nos anos de 1930, alguns resultados mostraram que as deficiências funcionais estavam relacionadas com a extensão do dano no córtex e não com a localização do dano [5].

Atualmente, métodos da neuropsicologia cognitiva são usados para identificar módulos de processamento mental na avaliação de pacientes com danos cerebrais. O dano cerebral é interpretado em termos da perda de um específico componente ou da desconexão entre componentes. Se um aspecto no desempenho de um paciente é deficiente, enquanto em outros é preservado, isso sugere a presença de um módulo de processamento separado. Tal abordagem para a investigação dos sistemas cognitivos produz métodos de avaliação para funções debilitadas que não são ligadas a áreas cerebrais particulares, mas sim, a componentes de habilidades e capacidade funcionais no cérebro.

O modelo neuropsicológico, assim, permite explicar as alterações comportamentais e cognitivas de cada novo paciente, constituindo um diagnóstico cientificamente fundamentado que constitui, por sua vez, uma contribuição valiosa para o diagnóstico neurológico. Além disso, permite estabelecer sobre bases científicas um programa de reabilitação baseado na identificação do componente do sistema que apresenta o déficit e o estado dos demais componentes do sistema suscetíveis a participar do processo de reabilitação.

\section{Reabilitação cognitiva e a reabilitação neuropsicológica}

Nos últimos anos, a reabilitação cognitiva passou por uma evolução no que concerne ao paradigma da intervenção, levando o modelo-padrão de tratamento da década de 1980, no qual "o terapeuta atuava individualmente fazendo avaliação cognitiva e treinamento de remediação cognitiva" [6] para uma intervenção mais abrangente e compreensiva: a reabilitação neuropsicológica (RN).

A reabilitação cognitiva envolve a reaprendizagem de habilidades cognitivas e a elaboração de estratégias de tratamento [7]. Comumente, após uma lesão no sistema nervoso central, com sequelas moderadas ou graves estão presentes comprometimentos das funções cognitivas, que podem levar à pouca ou nenhuma independência funcional $[1,6,8,9]$. Desse modo, a reabilitação neuropsicológica compreende um conjunto de estratégias de intervenção ou técnicas que tentam ajudar os pacientes a reduzir, controlar ou lidar com tais alterações. Com esse intuito, as intervenções neuropsicológicas visam a melhorar funcionalmente as capacidades deficitárias para aumentar a autogestão e independência do sujeito [10].

Na perspectiva da reabilitação neuropsicológica, uma lesão cerebral, além das consequências do tipo cognitiva, pode resultar em alterações do tipo comportamental, emocional e psicossocial. Essa é capaz de oferecer um panorama holístico sobre o indivíduo, pois, apesar dos déficits físicos e sensoriais serem os mais evidentes, há um comprometimento no desempenho das atividades cotidianas e produtivas, bem como nas relações interpessoais e, consequentemente, na sua qualidade de vida [11].

A reabilitação neuropsicológica deve ser direcionada às mudanças que melhorem as funções importantes para a rotina diária do indivíduo, beneficiando sua qualidade de vida. Para isso, são elaborados exercícios de reabilitação que geralmente são apoiados por formulários impressos, vídeos, CDs de áudio ou qualquer outro meio capaz de representar situações do cotidiano, nas quais o paciente é incentivado a se concentrar, interagir, raciocinar, tomar decisões, entender o discurso corrente e expressar sentimentos e pensamentos [12].

É nesse cenário que os recursos informatizados para a prática de reabilitação têm ganhado força, desde o final do século XX, apresentando bons resultados. A vantagem da utilização desse recurso se dá em razão de que o computador permite construir e apresentar itens que não são acessíveis no formato impresso, estruturando situações que se assemelham à vida cotidiana. Um software, por exemplo, pode ser elaborado para permitir uma grande variedade de materiais para a estimulação, como imagens em movimento e interativas, o que pode facilitar a construção de instruções de grande qualidade para aumentar a compreensão da tarefa e a motivação do sujeito. Outra vantagem, para a utilização dos sistemas computacionais, é a capacidade adaptativa do instrumento, que permite ao terapeuta aplicar tarefas de dificuldade correspondente ao nível de aptidão do sujeito. A utilização de sistemas informatizados na reabilitação abre as portas da interação humano-computador (IHC) e, portanto, situa o computador não como um simples intermediário, mas como um instrumento conceitual novo para formação e aplicação de técnicas de reabilitação. 


\subsection{Reabilitação neuropsicológica e a interação humano-computador}

O computador é o instrumento mais adequado para auxiliar qualquer processo de reabilitação cognitiva e é, também, extremamente útil para uma intervenção neuropsicológica, pois é capaz reunir todas as ferramentas necessárias para a apresentação de situações da vida diária e exercícios mais interativos [13]. O campo que se dedica a estudar os fenômenos de comunicação entre pessoas e sistemas computacionais é o da interação humano-computador.

A interseção das ciências da computação e informação às ciências sociais e comportamentais busca envolver todos os aspectos relacionados com a interação entre usuários e sistemas no intuito de fornecer explicações e previsões para fenômenos de interação usuário-sistema e resultados práticos para o projeto da interação [14]. Conforme esses autores, para garantir a aceitabilidade de um sistema, é necessário levar em consideração uma série de fatores humanos: (1) Fatores organizacionais: treinamento, políticas, etc; (2) Fatores ambientais: barulho, aquecimento, ventilação, luminosidade, etc.; (3) Saúde e segurança: estresse, dores de cabeça, perturbações musculares, etc.; (4) Conforto e localização: posição física, layout do equipamento, etc.; (5) Usuário: capacidade e processos cognitivos, motivação, satisfação, personalidade, experiência, etc.

Essa junção de disciplinas deu origem ao que chamamos de neurociência computacional (NC), a qual, apesar de ser recente, está crescendo virtuosamente, pois usa abordagens teóricas de uma variedade de disciplinas, incluindo matemática, física, ciência da computação e engenharia para entender o cérebro. A NC integra experiência, análise de dados e modelagem. Além disso, faz uso de uma linguagem científica que pode ser usada em todas as disciplinas e níveis de neurobiologia, ciência cognitiva e tecnologia da informação. É nesse nível que a neuropsicologia alia-se à neurociência computacional, podendo, assim, ajudar a resolver questões antigas de pesquisa, contribuindo para uma melhor prevenção e estratégias de tratamento para distúrbios neurais. Esses conceitos unificados sobre os processos biológicos e cognitivos, junto ao avanço das tecnologias da informação e interação homem-máquina, proporcionam uma nova visão para a concepção de estratégias eficientes para o ensino e aprendizagem.

Ao priorizar o olhar holístico sobre o homem, e não apenas as funções isoladas, a reabilitação neuropsicológica apresenta processos que, por meio de simulação em computador, fornecem uma base teórica e tecnologia que melhoram a nossa compreensão da função do comportamento. A reabilitação neuropsicológica com a utilização do computador é um campo de estudo emergente que demonstra a vantagem desse olhar para a compreensão do funcionamento da plasticidade cerebral durante o processo de reabilitação. Atualmente, a utilização dos computadores na avaliação e reabilitação neuropsicológica é baseada em três características: 1) a possibilidade de apresentações múltiplas, visuais e auditivas; 2) a capacidade para realizar medidas precisas, permitindo a avaliação da correção ou não das respostas, e a mensuração do tempo de reação; 3) a capacidade para armazenar e processar os dados, com a possibilidade subsequente de uma rápida avaliação e interpretação. Assim, a reabilitação neuropsicológica informatizada está preocupada com o design, a avaliação e a implementação de sistemas computacionais interativos para uso humano e com o estudo dos principais fenômenos ao redor desses, tendo como objetivo o conceito da aceitabilidade de um sistema (combinação de sua aceitabilidade social e sua aceitabilidade prática), denominado por Jakob Nielsen. Outro conceito importante da IHC para a reabilitação neuropsicológica é o de usefulness, que se refere à utilização do sistema para atingir um determinado objetivo, sendo uma combinação de utilidade e usabilidade. A utilidade deve verificar se a funcionalidade do sistema faz o que deve ser feito e a usabilidade está relacionada a quão bem os usuários podem usar a funcionalidade definida [46].

Por esse motivo, a neuropsicologia tem demonstrado ser a ponte que une saúde, comportamento e tecnologia, criando uma divisão muito original e estimulante, o que ajuda a colocar esse campo na vanguarda das intervenções não invasivas.

\section{Método}

A pesquisa foi realizada no portal de periódicos e no banco de teses da Capes. Na primeira etapa, foram consideradas publicações entre os anos 2008 a 2013. Os descritores utilizados para a busca foram: reabilitação cognitiva $A N D$ computador e reabilitação neuropsicológica $A N D$ computador. Esses termos foram inseridos em português e inglês, resultando na identificação de 229 publicações.

Revista Brasileira de Computação Aplicada (ISSN 2176-6649), Passo Fundo, v. 6, n. 1, p. 60-70, abr. 201463 
Na segunda etapa, foram excluídas publicações que possuíam pelo menos um dos seguintes critérios: (1) ausência da informação sobre utilização recursos informatizados no resumo; (2) ausência da informação sobre déficits cognitivos ou comportamentais no resumo; (3) pesquisas com modelos animais.

\section{Resultados}

A análise foi realizada com 121 artigos que descreveram minimamente a utilização de sistemas computacionais associados a programas de reabilitação cognitiva e/ou neuropsicológica. Contudo, conforme pode ser observado nas Tabelas 1 e 2 , é gritante a diferença das publicações que utilizam o referencial holístico da reabilitação neuropsicológica . Isso gera uma subutilização das ferramentas computacionais nessa prática.

Tabela 1. Publicações envolvendo utilização do computador para a reabilitação neuropsicológica

\begin{tabular}{l|c}
\hline Nome do periódico & Publicações \\
\hline MEDLINE (NLM) & 9 \\
Cambridge Journals (Cambridge University Press) & 2 \\
Expert Reviews (Future Science) & 1 \\
SciVerse ScienceDirect (Elsevier) & 3 \\
\hline Total de publicações & 15 \\
\hline
\end{tabular}

A literatura especializada sobre reabilitação neuropsicológica com suporte do computador começou a se construir nos últimos dez anos, valendo-se, seu conteúdo, de técnicas mais sofisticadas, como a realidade virtual. Contudo, são poucas as publicações, principalmente, devido à falta de investimento nesse cenário interdisciplinar e mais complexo.

Tabela 2. Publicações envolvendo utilização do computador para reabilitação cognitiva

\begin{tabular}{l|c}
\hline Nome do periódico & Publicações \\
\hline MEDLINE (NLM) & 73 \\
SciVerse ScienceDirect (Elsevier) & 41 \\
PMC (PubMed Central) & 21 \\
Cambridge Journals (Cambridge University Press) & 9 \\
SpringerLink & 8 \\
American Psychological Association (APA) & 6 \\
ERIC (U.S. Dept. of Education) & 5 \\
BioMed Central & 5 \\
Future Science Medicine & 1 \\
Oxford Journals (Oxford University Press) & 1 \\
nature.com (Nature Publishing Group) & 1 \\
\hline Total de publicações & 156 \\
\hline
\end{tabular}

O grande número de publicações com o tema de reabilitação cognitiva pode ter como principal motivo o desconhecimento da metodologia proposta pela neuropsicologia. Assim, novas pesquisas envolvendo a reabilitação neuropsicológica precisam ser realizadas, pois ainda são carentes no cenário científico nacional.

Tabela 3. Quantidade de publicações por década 1983-2013

\begin{tabular}{l|c}
\hline Ano de publicação & Publicações \\
\hline $1983-1993$ & 17 \\
$1994-2003$ & 43 \\
$2004-2013$ & 111 \\
\hline Total de publicações & 171 \\
\hline
\end{tabular}

Fica evidente a crescente produção de conhecimento envolvendo a utilização do computador nas práticas de reabilitação, provavelmente pela facilidade de acesso a esse equipamento atualmente. Um dos trabalhos pioneiros na área de reabilitação apoiada em computadores foi publicado por Bracy, em 1983, mostrando que o tempo decorrido após o dano cerebral é um dado que não pode ser desconsiderado, pois começar a terapia mais precocemente possível aumenta as chances de recuperação [15]. Na década de 1990, os computadores serviam 
basicamente como ferramentas para controlar a duração do estímulo apresentado, ainda sem grandes funções de interação. Contudo, a partir dessa década, a utilização de computadores foi impulsionada pelas novas tecnologias integradas aos procedimentos médicos, dentre as quais se destaca a utilização dos computadores para o treinamento e educação de pessoas portadoras de necessidades especiais, visando à melhoria de sua qualidade de vida [1].

Os programas de reabilitação até o final do século XX buscavam desenvolver as potencialidades físicas, mentais e sensoriais por meio da ajuda técnica de software. Especificamente, para a reabilitação cognitiva, verifica-se a disseminação de produtos que vão de programas simples, que atuam no tratamento de uma única função e exploram interfaces semelhantes, à prática tradicional [16].

Em 1997, Cunha utilizou o computador para estimular o processo de alfabetização funcional de pessoas diagnosticadas com síndrome de Down e obteve resultados positivos, criando um ambiente motivador para os pacientes que estavam reagindo ao processo de alfabetização tradicional. Outros pesquisadores apontam para o uso do computador no apoio a jovens com deficiência mental devido ao traumatismo crâneo-encefálico [17]. Esses autores exploraram os recursos sonoros do computador, associando-os a interfaces pictográficas, visando à reintegração desses indivíduos no ambiente escolar. Outros programas que facilitam a expressão de ideias e o florescimento da criatividade na produção de textos, em jovens com paralisia cerebral, também revelaram saltos qualitativos no processo de leitura/escrita [18].

Ainda no século XX, foi possível identificar a utilização do computador como recurso educacional com objetivos bastante específicos, que engloba tanto aspectos ligados ao desempenho físico, como ao desempenho intelectual. Nesses domínios, o computador emerge como ferramenta de alto potencial cognitivo e motivacional. Melo [19] ressalta que a possibilidade de promover o desenvolvimento cognitivo a partir do apoio da informática constitui-se uma das relações mais interessantes de se explorar entre a informática educativa e a psicologia, indo do paradigma comportamentalista a propostas mais abertas de construção do conhecimento.

Já no começo do século XXI, propostas mais sofisticadas que se apoiam em tecnologias promissoras como implementação de programas que utilizam ambientes virtuais: realidade virtual; realidade misturada; realidade aumentada; virtualidade aumentada; hiper-realidade; multimídia; dentre outras, começaram a ser utilizadas. Em 2001, a eficácia de um programa de remediação metafonológica e leitura, elaborado em versão computadorizada para escolares com dificuldades de aprendizagem foi provada evidenciando que houve diferença estatisticamente significativa entre a pré e pós-avaliação em todas as provas da versão avaliativa e em todas as habilidades trabalhadas na reabilitação [9]. Desse modo, os achados deste estudo mostram que os escolares com dificuldades de aprendizagem submetidos ao programa desenvolveram habilidades metafonológicas necessárias para o desenvolvimento da leitura.

Outro estudo [20], que evidencia a eficácia do programa de "Alfabetização fônica computadorizada" para intervenção de crianças e adolescentes com deficiência mental, mostra o aumento significativo do desempenho de leitura dos participantes, em média $20 \%$ superior no subteste de vocabulário da Escala de Inteligência Wechsler para crianças (WISC III). Também existem estudos [21,22,23] apontando para a eficácia dos programas de reabilitação em casos de neuroinfecções causadas pela malária e infecção congênita pelo HIV.

No que se refere à realidade virtual, entre os anos de 2005 e 2010, houve uma grande quantidade de trabalhos que discutiam sobre o uso da realidade virtual imersiva para a reabilitação cognitiva da síndrome disexecutiva, causada por lesões cerebrais pré-frontaais [24,25,26,27]. Os resultados expõem, no grupo que usou sistemas de realidade virtual imersiva, uma significativa melhora de respostas durante as tarefas, os dados neuropsicológicos mostram uma vantagem congruente da utilização do sistema de realidade virtual para a reabilitação. O estudo mostra que o grupo que realizou as atividades por meio do sistema computacional experimenta um aumento na ativação psicofisiológica, o que segundo os autores, pode ser interpretada de acordo com diferentes hipóteses: primeiro, essa ativação pode refletir maior participação emocional, na qual pode ser considerado um possível indicador de maior nível de atenção; em segundo lugar, pode, simplesmente, refletir um fator de estresse, relacionado ao fato de que as tarefas realizadas em uma condição não virtual poderiam ser mais difíceis ou cansativas [28,29].

Além de desordens relacionadas a lesões ou síndromes [41,42,43,44], já é possível identificar trabalhos relacionados a pessoas com desordens psiquiátricas e os ganhos cognitivos-comportamentais obtidos através do uso do computador [30,31,32,33,34,35,36]. Em um desses estudos [35] foram comparados dois grupos de pacientes com diferentes tipos de distúrbios e seu desempenho, tendo um dos grupos utilizado computadores 
para estimular a reabilitação, e o outro, as formas tradicionais. Os resultados obtidos indicaram que pacientes psiquiátricos podem trabalhar produtivamente com os computadores e que a reabilitação neuropsicológica assistida por computador pode produzir algumas melhoras no desempenho cognitivo e no repertório comportamental desses indivíduos.

\section{Conclusão}

Muitos dos artigos que foram excluídos não tratam de programa de reabilitação com utilização do computador, mas de métodos de avaliação. É possível identificar na literatura que há um número ascendente de publicações de validação de versões informatizadas de instrumentos de avaliação psicológica. Isso ocorre, pois, em geral, as experiências são realizadas por meio da utilização de sistemas tutores inteligentes cujo objetivo é garantir a aceitabilidade de um sistema pelo usuário, sendo considerados diversos fatores organizacionais (treinamento), ambientais (barulho), de saúde (estresse) e conforto (posição física). Esses fatores tão importantes na utilização de sistemas computacionais para a avaliação psicológica podem não ser o melhor caminho para a reabilitação neuropsicológica.

Conclui-se que a implementação de programas de reabilitação informatizados vem sendo desenvolvida apenas com base nos conhecimentos nosológicos, identificando as habilidades preservadas e prejudicadas. Esses estudos, em sua maioria, apresentam programas que realizam treinamento de habilidades e, muitas vezes, os aspectos socioemocionais não são levados em consideração e as questões ambientais, imprescindíveis para a reabilitação neuropsicológica, são ignoradas. Ao considerar todas essas informações, pontua-se que é possível fazer uso do computador de maneira mais ampla e assertiva, promovendo e facilitando a interação e inclusão do indivíduo ao seu ambiente.

Desse modo, manter o foco da utilização do computador na reabilitação cognitiva limita todo o potencial que essa ferramenta pode oferecer ao profissional. Esse, ao desenvolver um programa de reabilitação neuropsicológica informatizada, deve atentar para o fato de que cada etapa gera uma nova habilidade no paciente e essa deverá ser aplicada com sucesso em uma variedade de novos contextos. Isso requer o uso de estraté gias de memória e habilidades de pensamento, existindo diferentes abordagens para a reabilitação que se diferenciam a partir do critério conceitual utilizado. Assim, o profissional deve ter clareza da meta a ser alcançada. Nesse sentido, há alguns enfoques para guiar o objetivo do programa como restaurador, que utiliza a repetição sistemática e hierárquica para obter melhoria de desempenho dos componentes cognitivo e compensatório, visando ao desempenho de tarefas [47].

Sendo assim, podemos ultrapassar o paradigma de treinamento do processo cognitivo, como atenção e memória, buscando aliar essa prática a outras que focam no treinamento de habilidades funcionais, como a prática de um conjunto de passos em um trabalho de rotina. A seguir, podem ser observados, na Figura 1, alguns passos importantes para a elaboração e a análise de viabilidade de um programa informatizado de reabilitação.

\section{Figura 1 - Modelo de informatização do programa de reabilitação neuropsicológica}

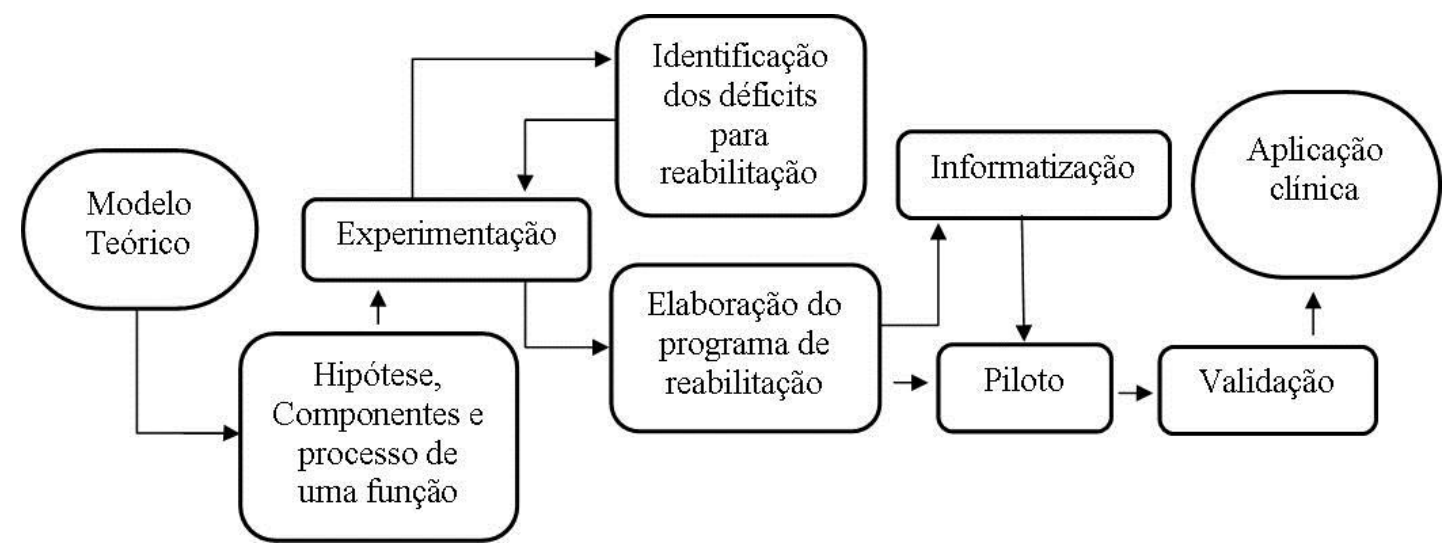

Uma característica importante no momento de realizar um plano para a reabilitação neuropsicológica é entender que a reabilitação deve ser centrada no sujeito e no ambiente, ou seja, se a pessoa tem capacidade de 
recuperar habilidades cognitivas ou se o meio em que vive terá que ser reestruturado para apoiar sua independência. A exploração de um desses enfoques não exclui a utilização de outro. Essas abordagens complementam-se e possibilitam a integração de vários aspectos essenciais para a recuperação do sujeito.

Nesse sentido, a utilização de ambientes virtuais na prática de reabilitação neuropsicológica deve ser considerada, enquanto parte do tratamento, uma ferramenta de apoio entre o trabalho realizado com o sujeito e o seu ambiente natural. Importante destacar que um indivíduo que está em reabilitação precisa de tarefas capazes de realizar a generalização e a transferência do aprendido em contexto de reabilitação para ser aplicado em outros contextos. A transferência pode ser positiva quando o que é aprendido em um contexto facilita o aprendido em outro, ou negativa quando o que é aprendido em um contexto impede o que é aprendido em outro, podendo ambas ser utilizadas pelo profissional a depender do objetivo da reabilitação [38,39,40,48].

Ainda podem ser ressaltados os aspectos associados a teorias pedagógicas como o comportamentalismo e construtivismo. Em geral, os processos de reabilitação neuropsicológica possuem o trabalho inicial comportamentalista e algumas vezes sem associações diretas com a vida diária. No entanto, para que haja generalização, é necessária a "exploração da memória, que realiza as atividades de recuperação de informações armazenadas, associação destas informações com a situação real, interpretação e análise deste conjunto de informações." [1]. Dessa maneira, mesmo com os futuros avanços dos ambientes virtuais para a reabilitação neuropsicológica, esses devem ser utilizados com maior ênfase na fase inicial do processo, entendendo ser fundamental, em um momento posterior, que a intervenção seja realizada nos ambientes cotidianos do sujeito, garantindo assim a adaptação do aprendido no setting terapêutico.

Esta pesquisa evidenciou que, nos últimos trinta anos de pesquisas e intervenções com a utilização do computador, apesar da disponibilidade de um amplo campo teórico e de ferramentas cada vez mais refinadas, não houve grandes avanços dentro do campo da reabilitação. Assim, os aspectos metodológicos apresentados neste trabalho sobre a reabilitação neuropsicológica com auxílio do computador são necessários para o desenvolvimento de ambientes específicos para esse fim, integrando as áreas de neurologia, neuropsicologia, informática, ciências cognitivas e educação, entre outras. Este trabalho visa estimular a elaboração de ferramentas e a formação de profissionais capazes de atuar nessa nova vertente da reabilitação [49]. Com as ferramentas computadorizadas, a neuropsicologia pode ir além da utilização da tecnologia para a reparação ou estimulação de funções cognitivas específicas, podendo agir, também, com mais qualidade e agilidade na correção de problemas de comportamento, na aquisição de novas habilidades e tratamento de várias psicopatologias. Um exemplo disso está no trabalho que vem sendo desenvolvido pelo nosso laboratório na confecção de testes de intervenções comportamentais por meio da internet com foco na insônia e modelagem comportamental para déficits atencionais. Ainda nessa área, já se encontram instrumentos que fazem uso de tecnologias de ponta em pesquisa e atendimento clínico, tais como o uso da internet e computadores de mão mantidos para coleta de dados em potencial para o diabetes mellitus tipo 1.

A expansão da utilização dos computadores para realização de intervenções e avaliações pela neuropsicologia parece ser o grande desafio desses tempos, pois colocar os novos recursos a serviço dos indivíduos é evidenciar outra face da tecnologia, melhorando a qualidade de vida dos pacientes, familiares e cuidadores. Por isso, se faz necessária a promoção de uma nova geração de cientistas treinados com habilidades interdisciplinares, que façam uso das neurociências e das ciências computacionais para o progresso das atividades de reabilitação.

\section{Referências}

[1] COSTA, R. M. E. M. Ambientes virtuais na reabilitação cognitiva de pacientes neurológicos e psiquiátricos, Tese D.Sc, Coppe Sistemas-UFRJ, Rio de Janeiro, 2000.

[2] BENEDET, M. J. Fundamento teórico y metodológico de la neuropsicología cognitiva. Madrid: Ministerio de Trabajo y Asuntos Sociales, 2002.

[3] RAO, S. Neuropsicological assessment. Neuropsichiatry. Eds. Fogel, Schiffer, Rao, 1996.

[4] RIZZO, A., BUCKWALTER, G.; NEUMANN, U. Virtual reality and cognitive rehabilitation: a brief review of the future, Journal of Head Trauma Rehabilitation, v. 12(6), p. 1-15, 1997.

[5] GRIEVE, J. Neuropsychology for occupational therapists, Blackwell Scientific Publications, 1993. 
[6] ABRISQUETA-GOMEZ, J. Reabilitação neuropsicológica: abordagem interdisciplinar e modelos conceituais na prática clínica. Porto Alegre, RS: Artmed, 2012.

[7] MCCOY, K. D. et al. Approaches to the cognitive rehabilitation of children with neuropsychological impairment, 1997.

[8] NOVELLI, M. M. P. C.; CANON, M. B. F. Avaliação da funcionalidade nos programas de reabilitação cognitiva. (Org.) Abrisqueta-Gomez, J. Reabilitação neuropsicológica: abordagem interdisciplinar e modelos conceituais na prática clínica. Porto Alegre: Artmed, 2012.

[9] CAPEllini, S. A., OliVEIRA, A. M.; PINHEIRO, F. H. Eficácia do programa de remediação metafonológica e de leitura para escolares com dificuldades de aprendizagem. Revista da Sociedade Brasileira de Fonoaudiologia, v. 16(2), 189-197, 2011.

[10] CICERONE, K. D.; et al. Evidence-based cognitive rehabilitation: Updated review of the literature from 1998 through 2002. Archives of Physical Medicine and Rehabilitation, Indianapolis, v. 86(8), 681-692, 2005

[11] CICERONE, K. D.; et al. Evidencebased cognitive rehabilitation: Recommendations for clinical practice. Arch Phys Med Rehabil, v. 8(1), 596-615, 1990.

[12] ANDRADE, A.; WAZLAWICK, R.; MARIANI, A. Metodologia para criação de roteiros educativos em realidade virtual. Revista Brasileira de Informática na Educação, v. 1(5), 69-76, 1999.

[13] COSTA, L. F.; RAMALHO, F. A. A usabilidade nos estudos de uso da informação: em cena usuários e sistemas interativos de informação. Perspectivas em Ciência da Informação, v. 15, n. 1, p. 92-117, 2010.

[14] BERTOTI, G. A.;OMAR, N. Informática na educação especial: tecnologias, sistemas computacionais e experiências realizadas na reabilitação cognitiva. Anais do XIII Simpósio Brasileiro de Informática na Educação - SBIE - UNISINOS, 2002.

[15] BRACY, O. Computer-based cognitive rehabilitation, Journal of Cognitive Rehabilitation. v. 1(1), 7-8, 1983.

[16] BROOKS, B. L. et al. Computerized neuropsychological testing to rapidly evaluate cognition in pediatric patients with neurologic disorders. Journal of Child Neurology, 27:982, 2012

[17] CORDEIRO, M. E.; SILVA, F. As ajudas técnicas na estimulação cognitiva de traumatizados crâneoencefálicos, IV Congresso RIBIE, Brasília, 1998.

[18] SANTAROSA, L.; MOORI, A.; FRANCO, B. Ambientes de aprendizagem computacionais como "Prótese" para o desenvolvimento de jovens portadores de paralisia cerebral. Revista Integração, MEC, v. 7(17), p. 33-40, 1996.

[19] MELO, H. Ambientes computacionales y desarrollo Cognitivo: perspectiva psicológica, Boletín de Informática Educativa, v. 2(2), p. 137-145, Colômbia, 1998.

[20] HEIN, J. M. et al. Leitura e consciência fonológica na deficiência mental: intervenções com o programa alfabetização fônica computadorizada. In: SENNYEY, A. L. et al. (Org.). Neuropsicologia e inclusão: tecnologias em (re)habilitação. São Paulo: Artes Médicas, 2007.

[21] BANGIRANA, P. et al. Malaria with neurological involvement in Ugandan children: effect on cognitive ability, academic achievement and behavior. Malaria Journal, 2011, 10:334

[22] BANGIRANA, P. et al. Reliability of the Luganda version of the Child Behaviour Checklist in measuring behavioural problems after cerebral malaria. Child and Adolescent Psychiatry and Mental Health, 2009, 3:38.

[23] BOIVIN, M. J. et al. Neuropsychological benefits of computerized cognitive rehabilitation training in Ugandan children surviving cerebral malaria and children with HIV. BMC Proceedings 2008, 2(Suppl 1):P7

[24] PRIORE, C. L. et al. Experience with V-STORE: considerations on presence in virtual environments for effective neuropsychological eehabilitation of executive functions. Cyberpsychology \& Behavior, v. 6(3), p. 231$325,2003$.

[25] RIZZO, A.; BUCKWALTER, J. G. Virtual reality and cognitive assessment and rehabilitation: the state of the art; virtual reality in neuro-psycho-physiology. Holanda: Ed G. Riva, IOS Press, 1997. 
[26] SEABRA, A. G.; CAPOVILlA, F. C.; MACEDO, E. C. Alfabetização fônica computadorizada: fundamentação teórica e guia para o usuário. São Paulo, SP: Memnon, v. 1. 2010, 80 p.

[27] TEMPLE, E. et at. Neural deficits in children with dyslexia ameliorated by behavioral remediation: evidence from functional MRI. National Academy of Sciences of the United States of America, 2000.

[28] VELOSO, A. F.; LOPES, R. C. W.; MELLO, S. A. Informática, inteligência e dislexia. Tecnologia em (Re)Habilitação Cognitiva, 1998.

[29] WATANABE, M. K. F.; TSUKIMOTO, D. R.; TSUKIMOTO, G. R. Terapia ocupacional e o uso do computador como recurso terapêutico. Acta fisiátrica, v. 10(1): p. 17-20, 2003.

[30] COSTA, R. M. E. M. Ambientes virtuais na reabilitação cognitiva de pacientes neurológicos e psiquiátricos. 2000. 141 f. Programa de Pós-Graduação de Engenharia da Universidade Federal do Rio de Janeiro, Rio de Janeiro, 2001.

[31] COSTA, R. M. E. M.; CARVALHO, L. A. V.; ARAGON, D. F. Novas tecnologias na reabilitação cognitiva. Simposio de Informática en Salud, 2000. Argentina. Disponível em: <http://www.sis.org.ar/sis2000/tecnologias_reabilitacao.pdf>. Acesso em: 21 dez. 2011.

[32] KURTZ, M. Cognitive and social cognitive predictors of change in objective versus subjective quality-oflife in rehabilitation for schizophrenia. Psychiatry Research, 200, 102-107, 2012.

[33] KURTZ, M. Predictors of change in life skills in schizophrenia after cognitive remediation. Schizophrenia Research, 107, 267-274, 2009.

[34] KURTZ, M. Symptoms versus neurocognition as predictors of change in life skills in schizophrenia after outpatient rehabilitation. Schizophrenia Research 102, p. 303-311, 2008.

[35] MAHONEY, M. J. Human change processes: the scientific foundations of psychotherapy. New York: Basic Books, 1998.

[36] MURTHY, N. V. et al. Computerized cognitive remediation training for schizophrenia: An open label, multi-site, multinational methodology study. Schizophrenia Research, 139 (2012) 87-91.

[37] VENTURA, M. I. et al. Hemispheric asymmetries and prosodic emotion recognition deficits in Parkinson's disease. Neuropsychologia 50 1936-1945, 2012.

[38] CAMPOS, M. B.; SILVERIRA, M. S. Tecnologias para a educação especial. Anais do IV Congresso da Rede Iberoamericana de Informática Educativa. Brasília, 1998. Disponível em: 〈http://www.c5.cl/ieinvestiga/ribie98.htm>. Acesso em: 04 jan. 2012.

[39] CERTIC da UTAD; Centro de engenharia de reabilitação em tecnologias de informação e comunicação. Disponível em: em <http://mecbraille.utad.pt/braille.html>. Acesso em: 07 jan. 2012.

[40] CHEN, S. H. et al. The effectiveness of computer-assisted cognitive rehabilitation for persons with brain injury, Brain Injury, v. 21(3), 197-209, 2007.

[41] BURDA, P., et al. Computer-assisted cognitive rehabilitation of chronic psychiatric impatients. Computers in Human Behavior, v. 10(3), 359-368, 1994.

[42] CUNHA, L. O uso do computador na alfabetização de portadores de Síndrome de Down. AQUILA, n. 2, Universidade Veiga de Almeida, p. 9-48, 1997.

[43] KATZ, R. C.; WERTZ, R. T. The efficacy of computer-provided reading tratament for chronic aphasic adults, Journal Speech Language Hearing Research, v. 40(3), p. 493-507, 1997.

[44] NIELSEN, J. Klinefelter's syndrome: an orientation. 2. ed. Denmark: Novo Nordisk A/S, 1991.

[45] PIZZAMIGLIO, L. et al. The use of optokinetic stimulation in rehabilitation of the hemineglect disorder. Cortex, 40, 441-450, 2004.

[46] ROCHA, H. V.; BARANAUSKAS, M. C. C. Design e avaliação de interfaces humano computador, 2011. 
[47] OMAR, N.; BERTOTI, G. A. Informática na educação especial: tecnologias, sistemas computacionais e experiências realizadas na reabilitação cognitiva. Anais do XIII Simpósio Brasileiro de Informática na Educação - SBIE - UNISINOS 2002.

[48] KAWASAKI, E. I. et al. Um modelo de sistema de tutoria inteligente baseado em princípios pedagógicos para a educação de adultos. SBIE 2000.

[49] CASSIDY, J.; EASTON, M.; CAPPELLI, C. Cognitive remediation on persons with severe and persistent mental ilness, Psychiatric Quartely, v. 67(4), 313-321, 1996.

[50] BOND, M. R. Assesment of psychological outcome after severe head injury. In: PORTER, R.; FITZSIMONS, W. (Org.). Outcome of severe damage to the central nervous system. Amsterdam: Elsevier, $\mathrm{p}$. 141-157, 1975. 